\title{
Prediction of RNA secondary structure with pseudoknots using coupled deep neural networks
}

\author{
Kangkun Mao' ${ }^{1}$, Jun Wang ${ }^{1}$, Yi Xiao ${ }^{1 凶}$ \\ 1 School of Physics and Key Laboratory of Molecular Biophysics of the Ministry of Education, Huazhong University of \\ Science and Technology, Wuhan 430074, China
}

Received: 12 June 2020 / Accepted: 3 July 2020 / Published online: 4 August 2020

\begin{abstract}
Noncoding RNAs play important roles in cell and their secondary structures are vital for understanding their tertiary structures and functions. Many prediction methods of RNA secondary structures have been proposed but it is still challenging to reach high accuracy, especially for those with pseudoknots. Here we present a coupled deep learning model, called 2dRNA, to predict RNA secondary structure. It combines two famous neural network architectures bidirectional LSTM and U-net and only needs the sequence of a target RNA as input. Benchmark shows that our method can achieve state-of-the-art performance compared to current methods on a testing dataset. Our analysis also shows that 2dRNA can learn structural information from similar RNA sequences without aligning them.
\end{abstract}

Keywords RNA secondary structure prediction, Deep learning, Minimum free energy

\section{INTRODUCTION}

RNAs participate in many important biological activities (Xiyuan et al. 2017; Zhao et al. 2016). To do these, they need to form correct tertiary structures in general. Therefore, it is necessary to know the tertiary structures of RNAs to understand their functions. At present, experimental determination of RNA tertiary structures are more difficult than proteins and so many theoretical or computational methods have been proposed to predict RNA tertiary structures (Cao and Chen 2011; Das et al. 2010; Jain and Schlick 2017; Wang et al. 2017; Wang and Xiao 2017; Xu et al. 2014). Although these methods use different principles, their performances all depend on the accuracy of RNA secondary structures. Therefore, accurate prediction of RNA secondary structure is very important.

Electronic supplementary material The online version of this article (https://doi.org/10.1007/s41048-020-00114-x) contains supplementary material, which is available to authorized users.

$\bowtie$ Correspondence: yxiao@hust.edu.cn (Y. Xiao)
Traditional methods of RNA secondary structure prediction can be divided mainly into two categories: single-sequence methods and homologous sequences methods. The single-sequence methods only need the sequence of target RNA as input and most of them are based on thermodynamic model or minimum free energy principle (Bellaousov et al. 2013; Janssen and Giegerich 2015; Zuker 2003). Therefore, the accuracy of prediction results of these methods depends largely on the thermodynamic parameters that are difficult to determine accurately (Zhao et al. 2018). Comparing to the single-sequence method, homologous sequence method uses the evolution information of homologous sequences to infer their common secondary structure (Lorenz et al. 2011), e.g., TurboFold (Tan et al. 2017). It was shown that the homologous sequences method usually could achieve higher accuracy than the singlesequence method (Puton et al. 2013). But the disadvantage of the homologous sequence method is also obvious, i.e., many RNAs of interest don't have sufficient numbers of homologous sequences at present, leading to low prediction accuracy using the homologous 
sequence method. Furthermore, correct prediction of pseudoknots is still a challenge for both methods.

Deep learning has recently showed impressive applications across a variety of domains. Very recently deep learning has also been applied to RNA secondary structure prediction, e.g., DMFold (Wang et al. 2019), which first predicts the dot or bracket state by bidirectional long short-term memory (LSTM) neural network and then infers the secondary structure by an algorithm based on improved base pair maximization principle. The input of DMFold is just the sequence of the target RNA. We also proposed to apply fully convolutional network (FCN) to improve RNA secondary structure prediction using direct coupling analysis of aligned homologous sequences of the target RNA (He et al. 2019). These methods show higher prediction accuracy than current related methods.

Here we propose a method of RNA secondary structure prediction with pseudoknots using coupled deep learning neural networks, which combines two famous neural networks architecture bidirectional LSTM (Krizhevsky et al. 2017) and U-net (Ronneberger et al. 2015). We call this method as 2dRNA. The sequence information of a target RNA is the only need for 2dRNA. Benchmarks show that 2dRNA can achieve state-of-theart performance compared to most popular prediction methods on a testing dataset.

\section{RESULTS}

In this section, 2dRNA is benchmarked on the two testing sets and its performance is compared with several existing methods, including Mfold (Zuker 2003), RNAfold (Zuker and Stiegler 1981), cofold (Proctor and Meyer 2013), Ipknot (Sato et al. 2011), Probknot (Bellaousov and Mathews 2010), and DMfold (Wang et al. 2019). Among them, Mfold, RNAfold and cofold could predict pseudoknot-free secondary structures, while Ipknot, Probknot and DMfold could predict secondary structures with pseudoknots. During preparing our manuscript, Zhou's group reported a method of RNA secondary structure prediction using deep learning and transfer learning that also achieved significantly higher prediction precision than current methods (Singh et al. 2019). Since they used a different dataset for training, it is inappropriate to compare the performance of their method with ours directly. So, we shall not compare them here but this issue will be discussed later in more detail in the next section.

Table 1 shows the prediction results of our method and other methods on the Archivell testing set. The prediction results of other methods are from previous
Table 1 The comparison between 2dRNA and other methods on the ArchiveII testing set

\begin{tabular}{llll}
\hline Method & $S T Y$ & $P P V$ & $M C C$ \\
\hline Mfold & 0.6658 & 0.6265 & 0.6459 \\
RNAfold & 0.5888 & 0.5290 & 0.5581 \\
Cofold & 0.5203 & 0.4883 & 0.5040 \\
IPknot & 0.5805 & 0.6163 & 0.5981 \\
Probknot & 0.5868 & 0.5285 & 0.5569 \\
DMfold & 0.7598 & 0.8585 & 0.8076 \\
2dRNA & 0.9017 & 0.9820 & 0.9353 \\
2dRNA + DB & 0.9288 & 0.9838 & 0.9519 \\
2dRNA $(<80 \%)$ & 0.8168 & 0.9631 & 0.8781 \\
2dRNA + DB $(<80 \%)$ & 0.8777 & 0.9787 & 0.9197 \\
\hline
\end{tabular}

The prediction results of other methods other than 2dRNA are from previous work (Wang et al. 2019)

work (Wang et al. 2019). It could be obviously seen that the STY, PPV and MCC values of 2dRNA far exceed other methods. See the supporting material for the detailed performance of our method on the testing set (Supplementary Table S1).

The output of 2dRNA is a contact map, which also includes pseudoknot information. In the Archivell testing set there are 72 structures with total 996 pseudoknot base pairs. For comparison, we chose IPknot (Sato et al. 2011) and Probknot (Bellaousov and Mathews 2010), the most famous ones that can predict pseudoknots. The result is shown in Table 2 where the number of true positive (TP) is in relative to the total 1114 pseudoknot base pairs and the same is for TN, FP, FN, STY, PPV and MCC. As it can be seen, 2dRNA has a much higher performance in pseudoknot base pairs than other methods, with MCC 0.650. After applying data balancing technique, the prediction accuracy can be further promoted and $M C C$ is up to 0.745 , even for a trained model $(2 \mathrm{dRNA}+\mathrm{DB}(<80 \%))$ with training data that only keep the sequences having similarity less than $80 \%$ with the testing set (see the following).

\section{DISCUSSION}

\section{Better prediction using data balancing technique}

The original training dataset contains total 2111 sequences with length less than 500 . Among them, most sequences (1390) are less than 200 in length, accounting for $65.8 \%$ of the total. Specifically, there are 436 sequences with length less than 100, 954 in 100-200, 92 in 200-300, 565 in 300-400, 64 in 400-500. The 
Table 2 Evaluation of pseudoknot prediction

\begin{tabular}{lrrlllll}
\hline Method & \multicolumn{1}{c}{$T P$} & $F P$ & $T N$ & $F N$ & $S T Y$ & $P P V$ & $M C C$ \\
\hline IPknot & 75 & 295 & 3415 & 924 & 0.071 & 0.125 & 0.089 \\
Probknot & 16 & 234 & 3436 & 983 & 0.011 & 0.035 & 0.018 \\
2dRNA & 584 & 47 & 4611 & 415 & 0.582 & 0.762 & 0.650 \\
2dRNA + DB & 726 & 62 & 5088 & 273 & 0.706 & 0.809 & 0.745 \\
2dRNA (<80\%) & 269 & 88 & 3334 & 730 & 0.169 & 0.373 & 0.242 \\
2dRNA + DB $(<80 \%)$ & 597 & 38 & 4495 & 402 & 0.600 & 0.735 & 0.653 \\
\hline
\end{tabular}

number of the long RNA sequences is so few that our model can't learn enough knowledge from it for dealing with long sequence prediction. So here we apply the data balancing technique, not increasing new data but just duplicating the minority long sequences to let our model train with those data more times. In particular, we have duplicated the sequences with length in $200-300$ by six times, in $400-500$ by nine times, bring about total 3083 sequences on balanced training set. Figure $3 \mathrm{~A}$ and $\mathrm{B}$ illustrate the distribution before and after data balancing.

After this data processing, our model can well deal with long RNA sequences, and the whole accuracy on testing set is also improved a lot. The $S T Y$ has increased by approximately $3 \%$ from 0.9017 to 0.9288 , while $P P V$ has just improved a little from 0.9820 to 0.9838 , resulting in the highest MCC 0.9519 among all the other methods. Especially for the long sequences, the prediction results look much better than before (Fig. 1C).

\section{Correlation between similarity and accuracy}

As it is well known that deep learning is a black box, making it hard to understand what it has learned. Here we try to give some insights about how prediction accuracy is correlated with the sequence similarity between the testing and training sets.

We used the Levenshtein distance (Levenshtein 1966) to measure the similarity between two sequences. It is noted that this kind of similarity is different from that given by CD-HIT-EST algorithm (Fu et al. 2012). For each sequence in the testing set, its distances with every sequence in the training set are calculated. Figure 2 shows the correlations between the prediction accuracy (here MCC) of each sequence in the testing set and the number of the sequences in the training set with their distances being larger than equal to 0.7 to the former. It shows that the prediction accuracy increases with the number of the sequences in general. For shorter sequences (blue dots in Fig. 2), the MCCs of most of them are close to 1 . This means that their structural information could be learned from those sequences with lower similarity. For the longer sequences (e.g., $>300$ (red dots in Fig. 2)) in the testing set, they usually have lower accuracy than the shorter ones with the same number of similar sequences. This is reasonable because for long sequences, more homologous sequences are needed to learn their structural information. These results suggest that the neural network model can learn common structural features from
A

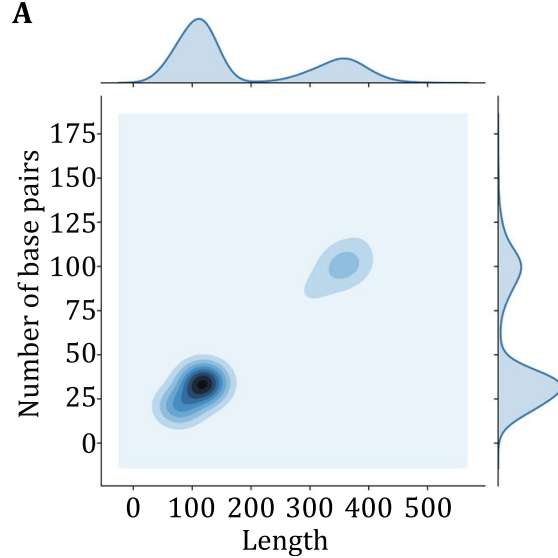

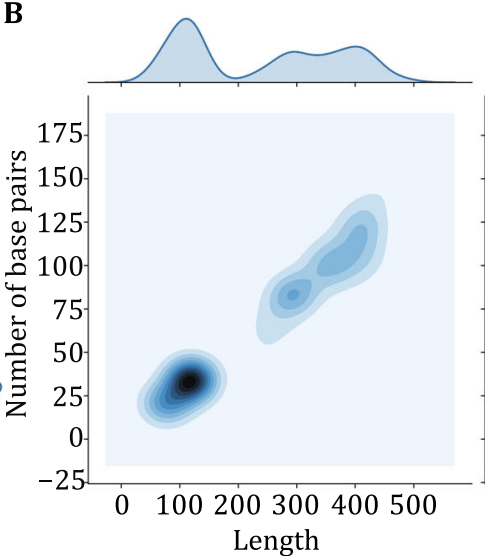

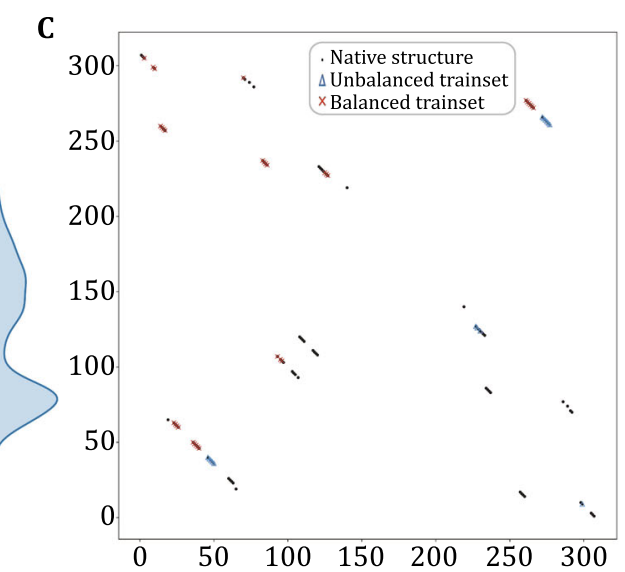

Fig. 1 Comparison of using data balancing technique or not. A The distribution of sequences length and number of base pairs on unbalanced training set. B Training set distribution after data balancing. C The result of RNaseP_Onchorhynchus-2. The black points are the native base pairs. The red is the prediction result of 2dRNA trained on balanced training set, and the blue is the prediction result of 2dRNA trained on unbalanced training set 
Fig. 2 Correlation between accuracy (here MCC) of each sequence in the testing set and the number of the sequences in the training set with their distances being larger than or equal to 0.7 to the former. The sequence length is scaled in color

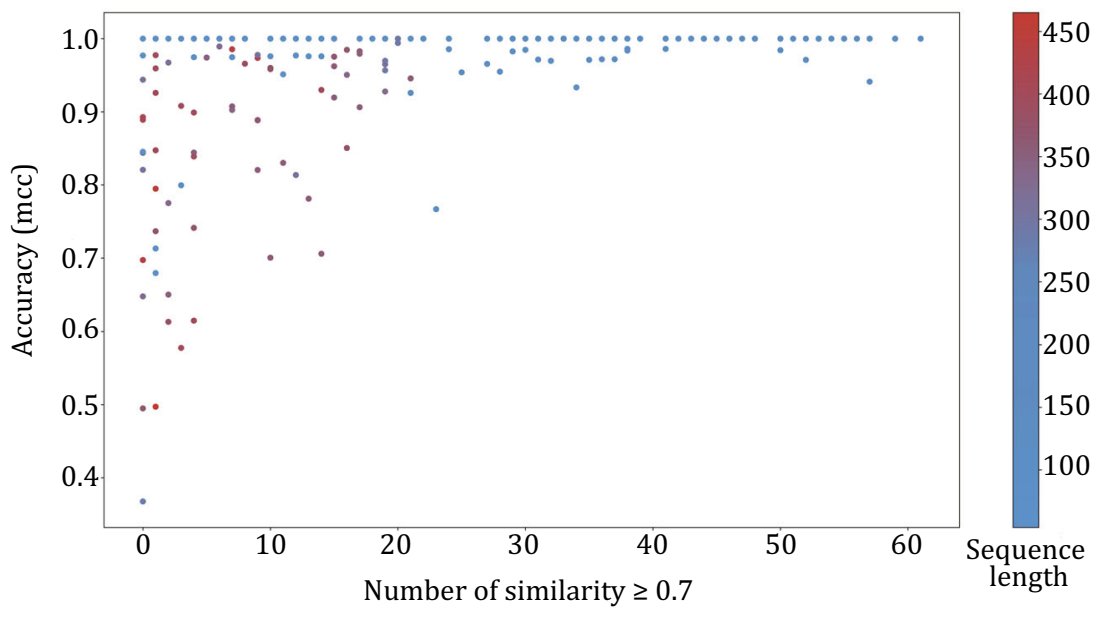

similar RNA sequences without aligning them. This is in agreement with previous study (Wang et al. 2019).

To see the generalization ability of 2dRNA, we also have trained a new model with training data that only keep the sequences having similarity less than $80 \%$ with the testing set using the CD-HIT-EST (sequenceidentity cutoff is set as 0.8 ) (Fu et al. 2012). The result is shown in Table 1 as 2dRNA $(<80 \%)$ and $2 \mathrm{dRNA}+\mathrm{DB}$ $(<80 \%)$. It is clear that in this case 2dRNA still significantly outperforms other methods in PPV, STY and MCC.

\section{Performance for different RNA types}

Table 3 shows the performances of 2dRNA for different types of RNAs in the testing set. The performances for 5sRNA and tRNA are higher than tmRNA and RNAseP, mainly in STY. This may be that the latter two usually have much longer lengths than the former in the training set (Supplementary Table S2). Furthermore, as discussed above, the sequence number of longer lengths is much small and this may be also a reason why the performance for tmRNA and RNAseP is relatively lower.

Our method also has been tested on the Zhou's testing set TS0 (38), which contains 1305 sequences

Table 3 Performance of 2dRNA for different RNA types on ArchivelI testing set

\begin{tabular}{lclll}
\hline Type & Number & $S T Y$ & $P P V$ & $M C C$ \\
\hline 5sRNA & 109 & 0.9898 & 0.9912 & 0.9903 \\
tRNA & 50 & 0.9736 & 0.9900 & 0.9804 \\
tmRNA & 31 & 0.7723 & 0.9601 & 0.8544 \\
RNaseP & 44 & 0.8369 & 0.9754 & 0.8931 \\
\hline
\end{tabular}

that include not only the four types mentioned above but also microRNA, riboswitch and so on. Table 4 shows the performances of 2dRNA for different types of RNAs in the TS0 testing set. Since our training dataset does not contain these other types of RNAs, it's not surprising to see poor performance of those. But in 5sRNA, tRNA, tmRNA and RNaseP, our method still maintains impressive performance.

\section{CONCLUSION}

In this work, we present an end-to-end coupled deep learning model 2dRNA to predict RNA secondary structure with pseudoknots. Our method achieves a state-of-the-art performance compared to other methods, including the prediction of pseudoknots. It seems

Table 4 Performance of 2dRNA for different RNA types on TS0 testing set

\begin{tabular}{lrlll}
\hline Type & Number & $S T Y$ & $P P V$ & $M C C$ \\
\hline 5sRNA & 30 & 0.6807 & 0.8028 & 0.7321 \\
tRNA & 128 & 0.8761 & 0.9463 & 0.9058 \\
tmRNA & 25 & 0.6807 & 0.9042 & 0.7747 \\
RNaseP & 23 & 0.7742 & 0.8501 & 0.8001 \\
snRNA & 148 & 0.1335 & 0.1438 & 0.1272 \\
MicroRNA & 202 & 0.0627 & 0.2463 & 0.1177 \\
Riboswitch & 48 & 0.0169 & 0.0826 & 0.0327 \\
Group I Introns & 10 & 0.0277 & 0.4141 & 0.0992 \\
Group II & 55 & 0.0080 & 0.1091 & 0.0287 \\
CRISPR & 7 & 0.0612 & 0.1071 & 0.0810 \\
Other & 629 & 0.0586 & 0.1696 & 0.0897 \\
\hline
\end{tabular}


that the coupled deep learning model can use structural information learning from similar RNAs with known structures to predict the secondary structure of a target RNA. Our model was trained in the dataset with limited types of RNAs. In future we shall further apply it to larger dataset. Our method is available as a web server at https://biophy.hust.edu.cn/new/2dRNA. The web server is currently only for evaluating our method.

\section{MATERIALS AND METHODS}

\section{Problem formulation}

Here, the secondary structure of a RNA is defined as the pattern formed by the canonical base pairs A-U and C-G, and the wobble base pair G-U as usual. Non-canonical pairs are neglected in our secondary structure prediction. The bases in the secondary structure fulfill the following constraints: (1) a base cannot participate in more than one base pair; (2) bases that are paired with each other must be separated by at least three bases along sequence.

In this work, we used two different representations of RNA secondary structure. One is dot-bracket notation (Hofacker et al. 1994), which contains seven symbols. Unpaired nucleotides are indicated with the character "., matching pairs of parentheses " 0 " indicate base pairs. To indicate non-nested base pairs (pseudoknots), additional brackets are used: "[]", " \{\} ", or " $<>$ ". Another representation is the dot-plot matrix (Maizel and Lenk 1981), where each base pair $(i, j)$ is represented by a dot or box in row $i$ and column $j$ of a rectangular grid or contact matrix of the structure. Figure 3 shows the RNA secondary structure and its two representations.

\section{Methods}

Our deep learning neural network is a coupled twostage model: coarse-grained dot-bracket prediction (CGDBP) and fine-grained dot-plot prediction (FGDPP). Motivated by DMfold (Wang et al. 2019), CGDBP takes one-hot encoding format of four type bases of the sequence as input and predicts the dot-bracket states of the bases. We use a two-layer bidirectional LSTM (Krizhevsky et al. 2017) as the encoder that encodes the input sequence information into high dimension space (the dimension of the vectors is $L \times 256$, where $L$ is the length of input sequence, 256 is the encoding feature size), followed by a decoder to give dot-bracket prediction. This decoder is a fully connected network, taking the encoding vectors from RNA sequence to one-hot format of dot-bracket sequences $(L \times 7$, for the dotbracket notation contains seven symbols). The FGDPP is a fully convolution network, which inputs RNA sequence encoding but outputs dot-plot matrix $(L \times L)$. The output of first stage is the dot-bracket state of each base, i.e., the base is in unpaired state "." or in one of the six paired states “(", ")", “[”, "]", “\{" or "\}", which tells what state each base is at, but there is still much mismatching between brackets. That is why it's called coarse-grained prediction. The second part FGDPP of our model is created to address this problem. Since the LSTM network can only capture sequential context, we combined it with U-net (Ronneberger et al. 2015) to get precise pairwise base-pairing prediction and provide the ability of extracting structural information. Figure 4 displays the coupled neural network architecture of 2dRNA.

\section{Coarse-grained dot-bracket prediction}

The CGDBP uses the bidirectional LSTM architecture (Krizhevsky et al. 2017) as encoder and a fully connected layer as decoder. LSTM network (Hochreiter and
A

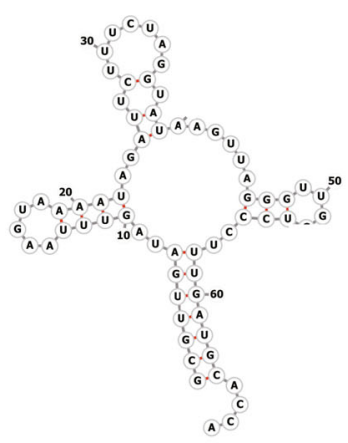

B

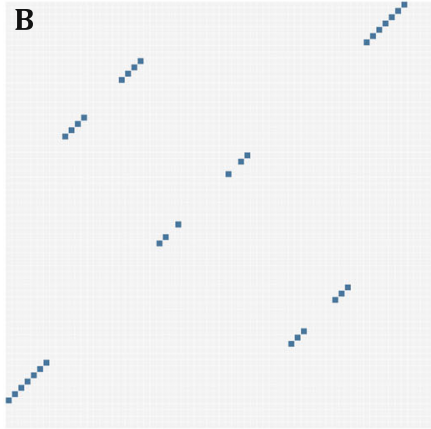

C

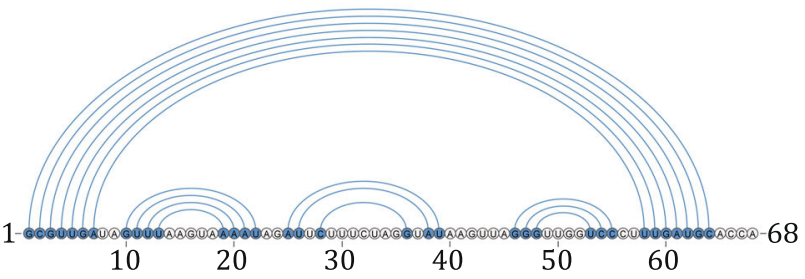

Fig. 3 RNA secondary structure and its two representations. The secondary structure of a RNA (A), its dot-plot matrix representation (B) and its dot-bracket representation (C) 


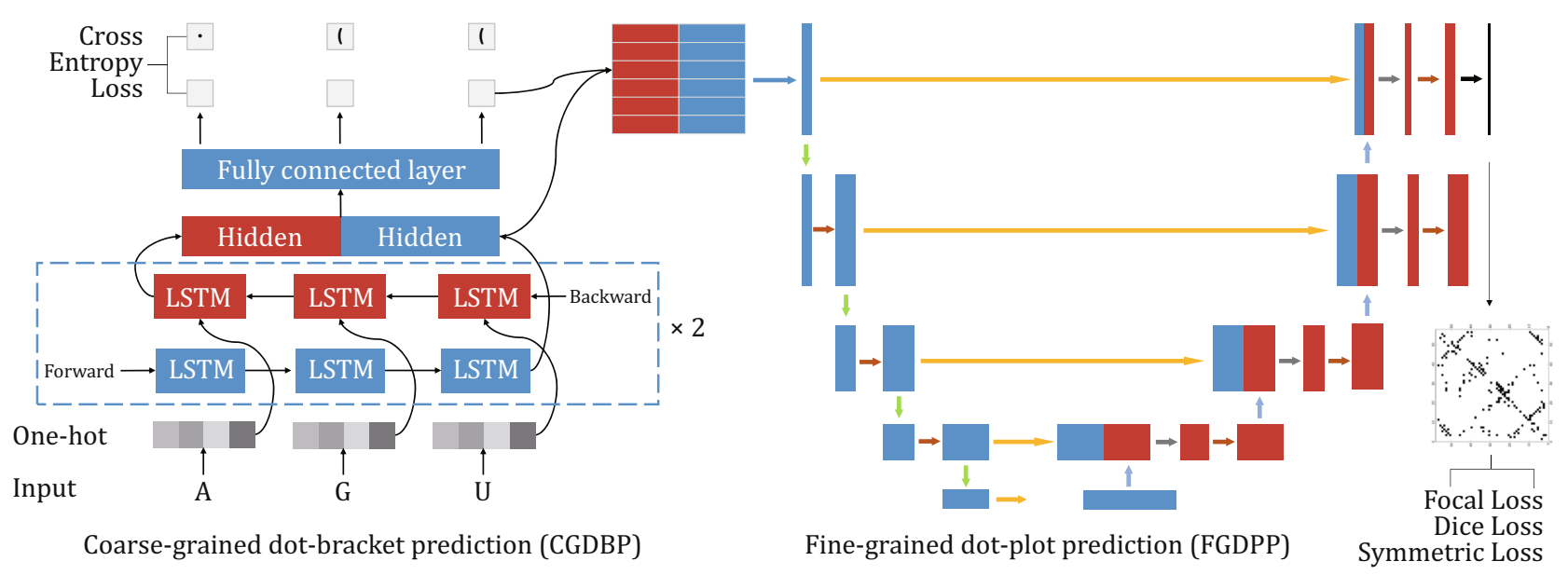

Fig. 4 The coupled neural network architecture of 2dRNA. Coarse-grained dot-bracket prediction uses two-layer bidirectional LSTM and a fully connected layer to output dot-bracket prediction and fine-grained dot-plot prediction uses fully convolutional network (U-net) to predict pairwise base-pairing as final result

Schmidhuber 1997) is a special kind of recurrent neural network, capable of learning long-term dependency and now is widely used in natural language processing. Our model uses a 2-layers bidirectional LSTM network, the dimension of hidden vector $h$ is 128 for all the layers. The decoder is a 3-layers fully connected network, with size of 128,64 and 7 . In both networks, the dropout rate is 0.1 and the activation function is rectified linear unit (ReLU) (Agarap 2018).

The input of CGDBP is RNA sequences, one-hot encoding by $L \times 4$ vectors. Thence the residue $\mathrm{A}$ is encoded as " 1000 ", $\mathrm{U}$ is " 0100 ", G is " 0010 ", and $\mathrm{C}$ is "0001". Then it outputs the $L \times 7$ vectors which correspond to the one-hot vector of dot-bracket symbols, with "0001000" represents "., " 1000000 " and "0000001" represent "(" and ")"; "0100000" and "0000010" for "[" and "]", and "0010000" and "0000100" for " " " and " $\}$ ".

\section{Fine-grained dot-plot prediction}

The FGDPP uses the U-net architecture (Ronneberger et al. 2015) as decoder. U-Net is a famous network developed for biomedical image segmentation, which is based on FCN (Shelhamer et al. 2017) and its architecture is extended to yield more precise segmentations. U-net consists of a contracting path for downsampling and an expansive path for upsampling. During the contraction path, the spatial information is reduced while feature information is increased. Compared to the vanilla fully convolutional networks, U-net has the shortcut connection crossing convolutional layers analogous to ResNet, its expansive path concatenates the spatial information with high-resolution features from the contracting path, which provide local information to the global. This is significant to the secondary structure problem, high-resolution features make a benefit to the local structures like stems or hairpins, global features about these local structures provide spatial information that contributes to the prediction of global structure, especially for pseudoknots.

We used a 9-blocks/layers U-net in our model. The contracting path is composed of four blocks, each block is composed of: (1) two units of $3 \times 3$ convolution layer and ReLU activation function (with batch normalization), followed by (2) $2 \times 2$ max pooling. The number of feature maps doubles at each pooling, starting with 32 feature maps for the first block to the final 256 feature maps. The parameters of all the convolution layers are the same, with kernel size being 3 , stride being 1 , and padding being 1 . The middle part of U-net between the contracting and expanding paths is a block built from simply two convolutional layers with dropout and batch normalization. And the last two blocks are the upsampling path, each of these blocks is composed of: (1) deconvolution layer with stride 2; (2) skip connections with the corresponding cropped feature map from the contracting path; (3) two $3 \times 3$ convolution layers and ReLU activation function (with batch normalization).

We take the output of the bidirectional LSTM, which is 256-dimensional encoding vectors, and do a pairwise addition operation on it to get a tensor with size $L \times L \times 256$, as the input of the U-net. The output of the $\mathrm{U}$-net is a $L \times L \times 1$ matrix, which is through the sigmoid layer to be normalized into $[0,1]$, reflecting the pairing probability of each pair of bases. In our model, 
we set the cutoff as 0.5 , the score of base pairs that exceed this cutoff is considered as paired.

The original U-net uses binary cross entropy loss to optimize its parameters (Goodfellow et al. 2016). But for RNA secondary structure prediction problem, the native base pair is so rare relative to all possible pairing in dot-plot matrix representation, i.e., positive labels are very lacking in training set, which cause inefficient training as most locations are simple negatives and contribute no useful learning signal. Mass negatives can overwhelm training and lead to degenerate models. So we used focal loss function (Lin et al. 2018) to address this class imbalance, which reshapes the standard cross entropy loss such that it down-weights the loss assigned to well-classified examples. Another loss function our model uses is soft dice loss, which is based on the dice coefficient (DSC) that is a measure of overlap between two samples (Milletari et al. 2016). Especially for RNA, we proposed a symmetric loss function according to its secondary structure. The RNA dot-plot representation is a symmetric matrix, but the predictions are often not. So, a symmetric loss as a penalty is used to prevent occurring of this:

$S L(p)=\left(p-p^{\top}\right)^{2}$,

Those two parts (coarse-grained and fine-grained) are trained together as a whole, for the reason that both dot-bracket output and matrix output can help and constrain each other in the process of loss propagation. While training, we used Adam (Kingma and Ba 2014) with learning rate 0.002 to optimize loss function. The train epoch is set to 30 , and batch size is 16 , since $L$ is different, forward calculate one sample at a time and then backward total loss after 16 samples, meanwhile the batch normalization in U-net is instance normalization. The PyTorch implement of U-net that we use comes from the open-source codes provided by milesial on Github (https://github.com/milesial/Pytorch-UNet).

\section{Data collection and processing}

The data used for training and testing contain sequence and secondary structure, is the same as DMFold (Wang et al. 2019) for the performance comparison. They are the ArchiveII dataset from the public database of Mathews lab (Ward et al. 2017). Although the RNA types of this dataset are less than the larger bpRNA dataset (Danaee et al. 2018), the sequence number of each RNA type has similar order of magnitude and so this dataset is better for training and testing a deep learning model (Supplementary Table S2). This dataset totally has 2345 known RNA primary sequences and their secondary structures. For comparing with DMFold, the same 234 sequences randomly chosen is taken as testing set. The left is for training and validating. Here we used tenfold cross-validation, split the left data into tenfolds, each time use ninefolds to train the model and onefold for validation. We have trained 10 models and used the averaged results of these models as final predictions, i.e., two residues are considered to form base pair if their averaged pairing probability is equal to or larger than 0.5 .

All data above are adopted to remove the duplicate data by the CD-HIT tool (Fu et al. 2012) and the data that contain the unknown bases. Particularly, we only selected the sequences with length less than 500 and longer than 32 . Finally, the training set contains totally 2111 sequences with length less than 500 .

\section{Performance metrics}

To estimate the performance of 2dRNA and other methods, we can calculate the accuracy of the base pairs to represent the accuracy of prediction structures. So that, the predictions can be compared between different methods. We use the precision (PPV) and sensitivity (STY) (Matthews 1975) to measure the performance of the methods. STY measures the ability to find the positive base pairs, while PPV measures the ability of not predicting false positive base pairs. They are defined as follows:

$P P V=\frac{T P}{T P+F P}$,
$S T Y=\frac{T P}{T P+F N}$,

where TP denotes true positive; FP, false positive; TN, true negative; $\mathrm{FN}$, false negative.

Generally, the requirements of PPV and STY could not be satisfied simultaneously when comparing the accuracy of the prediction results. Therefore, the Matthews correlation coefficients (MCC) (Parisien et al. 2009) is used to comprehensively evaluate the prediction results.

$$
\begin{aligned}
M C C & =\frac{T P \times T N-F P \times F N}{\sqrt{(T P+F P(F P+T N)(T N+F N)(F N+T P)}} \\
& \approx \sqrt{S T Y \times P P V} .
\end{aligned}
$$

Acknowledgements This work is supported by the National Natural Science Foundation of China (31570722). The data used for initial training along with their annotated secondary structure are publicly available at https://biophy.hust.edu.cn/new/2dRNA. 


\section{Compliance with Ethical Standards}

Conflict of interest Kangkun Mao, Jun Wang and Yi Xiao declare no conflict of interest.

Human and animal rights and informed consent This article does not contain any studies with human or animal subjects performed by any of the authors.

Open Access This article is licensed under a Creative Commons Attribution 4.0 International License, which permits use, sharing, adaptation, distribution and reproduction in any medium or format, as long as you give appropriate credit to the original author(s) and the source, provide a link to the Creative Commons licence, and indicate if changes were made. The images or other third party material in this article are included in the article's Creative Commons licence, unless indicated otherwise in a credit line to the material. If material is not included in the article's Creative Commons licence and your intended use is not permitted by statutory regulation or exceeds the permitted use, you will need to obtain permission directly from the copyright holder. To view a copy of this licence, visit http://creativecommons.org/ licenses/by/4.0/.

\section{References}

Agarap AFM (2018) Deep learning using rectified linear units (ReLU). arXiv: neural and evolutionary computing. https:// www.arxiv-vanity.com/papers/1803.08375

Bellaousov S, Mathews DH (2010) ProbKnot: fast prediction of RNA secondary structure including pseudoknots. RNA 16(10):1870-1880

Bellaousov S, Reuter JS, Seetin MG, Mathews DH (2013) RNAstructure: web servers for RNA secondary structure prediction and analysis. Nucleic Acids Res 41(W1):W471W474

Cao S, Chen SJ (2011) Physics-based de novo prediction of RNA 3D structures. J Phys Chem B 115(14):4216-4226

Danaee P, Rouches M, Wiley M, Deng D, Huang L, Hendrix D (2018) bpRNA: large-scale automated annotation and analysis of RNA secondary structure. Nucleic Acids Res 46(11):5381-5394

Das R, Karanicolas J, Baker D (2010) Atomic accuracy in predicting and designing noncanonical RNA structure. Nat Methods 7(4):291-294

Fu L, Niu B, Zhu Z, Wu S, Li W (2012) CD-HIT: accelerated for clustering the next-generation sequencing data. Bioinformatics 28(23):3150-3152

Goodfellow I, Bengio Y, Courville A (2016) Deep learning. MIT Press, Cambridge, MA

He X, Li S, Ou X, Wang J, Xiao Y (2019) Inference of RNA structural contacts by direct coupling analysis. Commun Inf Syst 19(3):279-297

Hochreiter S, Schmidhuber J (1997) Long short-term memory. Neural Comput 9(8):1735-1780

Hofacker IL, Fontana W, Stadler PF, Bonhoeffer LS, Tacker M, Schuster P (1994) Fast folding and comparison of RNA secondary structures. Monatshefte Fur Chemie 125(2):167-188

Jain S, Schlick T (2017) F-RAG: generating atomic coordinates from RNA graphs by fragment assembly. J Mol Biol 429(23):3587-3605
Janssen S, Giegerich R (2015) The RNA shapes studio. Bioinformatics 31(3):423-425

Kingma DP, Ba JA (2014) A method for stochastic optimization. arXiv: 1412.6980

Krizhevsky A, Sutskever I, Hinton GE (2017) ImageNet classification with deep convolutional neural networks. Commun ACM 60(6):84-90

Levenshtein VI (1966) Binary codes capable of correcting deletions, insertions and reversals. Sov Phys Dokl 10(4):707-710

Lin TY, Goyal P, Girshick R, He K, Dollar P (2018) Focal loss for dense object detection. IEEE Trans Pattern Anal Mach Intell. https://doi.org/10.1109/TPAMI.2018.2858826

Lorenz R, Bernhart SH, Siederdissen CHZ, Tafer H, Flamm C, Stadler PF, Hofacker IL (2011) ViennaRNA Package 2.0. Algorithms Mol Biol. https://doi.org/10.1186/1748-7188-626

Maizel JV Jr, Lenk RP (1981) Enhanced graphic matrix analysis of nucleic acid and protein sequences. Proc Natl Acad Sci USA 78(12):7665-7669

Matthews BW (1975) Comparison of the predicted and observed secondary structure of T4 phage lysozyme. Biochim Biophys Acta 405(2):442-451

Milletari F, Navab N, Ahmadi SA (2016) V-net: fully convolutional neural networks for volumetric medical image segmentation. Proceedings of 2016 Fourth International Conference on 3d Vision (3dv), 565-571

Parisien M, Cruz JA, Westhof E, Major F (2009) New metrics for comparing and assessing discrepancies between RNA 3D structures and models. RNA 15(10):1875-1885

Proctor JR, Meyer IM (2013) COFOLD: an RNA secondary structure prediction method that takes co-transcriptional folding into account. Nucleic Acids Res 41(9):e102. https:// doi.org/10.1093/nar/gkt174

Puton T, Kozlowski LP, Rother KM, Bujnicki JM (2013) CompaRNA: a server for continuous benchmarking of automated methods for RNA secondary structure prediction. Nucleic Acids Res 41(7):4307-4323

Ronneberger O, Fischer P, Brox T (2015) U-Net: convolutional Networks for Biomedical Image Segmentation. Med Image Comput Comput-Assist Interv 9351:234-241

Sato K, Kato Y, Hamada M, Akutsu T, Asai K (2011) IPknot: fast and accurate prediction of RNA secondary structures with pseudoknots using integer programming. Bioinformatics 27(13):i85-93

Shelhamer E, Long J, Darrell T (2017) Fully convolutional networks for semantic segmentation. IEEE Trans Pattern Anal Mach Intell 39(4):640-651

Singh J, Hanson J, Paliwal K, Zhou Y (2019) RNA secondary structure prediction using an ensemble of two-dimensional deep neural networks and transfer learning. Nat Commun 10:5407. https://doi.org/10.1038/s41467-019-13395-9

Tan Z, Fu YH, Sharma G, Mathews DH (2017) TurboFold II: RNA structural alignment and secondary structure prediction informed by multiple homologs. Nucleic Acids Res 45(20):11570-11581

Wang J, Xiao Y (2017) Using 3dRNA for RNA 3-D structure prediction and evaluation. Curr Protoc Bioinformatics. https://doi.org/10.1002/cpbi.21

Wang J, Mao K, Zhao Y, Zeng C, Xiang J, Zhang Y, Xiao Y (2017) Optimization of RNA 3D structure prediction using evolutionary restraints of nucleotide-nucleotide interactions from direct coupling analysis. Nucleic Acids Res 45(11):6299-6309

Wang L, Liu Y, Zhong X, Liu H, Lu C, Li C, Zhang H (2019) DMfold: a novel method to predict RNA secondary structure with pseudoknots based on deep learning and improved base pair 
maximization principle. Front Genet 10:143. https://doi.org/ 10.3389/fgene.2019.00143

Ward M, Datta A, Wise M, Mathews DH (2017) Advanced multiloop algorithms for RNA secondary structure prediction reveal that the simplest model is best. Nucleic Acids Res 45(14):8541-8550

Xiyuan L, Dechao B, Liang S, Yang W, Shuangsang F, Hui L, Haitao L, Chunlong L, Wenzheng F, Runsheng C, Yi Z (2017) Using the NONCODE database resource. Curr Protoc Bioinform. https:// doi.org/10.1002/cpbi.25

Xu X, Zhao P, Chen SJ (2014) Vfold: a web server for RNA structure and folding thermodynamics prediction. PLoS ONE 9(9):e107504. https://doi.org/10.1371/journal.pone. 0107504
Zhao Y, Li H, Fang SS, Kang Y, Wu W, Hao YJ, Li ZY, Bu DC, Sun NH, Zhang MQ Chen RS (2016) NONCODE 2016: an informative and valuable data source of long non-coding RNAs. Nucleic Acids Res 44(D1):D203-D208

Zhao Y, Wang J, Zeng C, Xiao Y (2018) Evaluation of RNA secondary structure prediction for both base-pairing and topology. Biophys Rep 4(3):123-132

Zuker M (2003) Mfold web server for nucleic acid folding and hybridization prediction. Nucleic Acids Res 31(13):3406-3415

Zuker M, Stiegler P (1981) Optimal computer folding of large RNA sequences using thermodynamics and auxiliary information. Nucleic Acids Res 9(1):133-148 